Supporting information

\title{
Doping Induced Orbit-orbit Interaction between Excitons while Enhancing Photovoltaic Performance in Tin Perovskite Solar Cells
}

Jia Zhang, Jiajun Qin, Ting Wu, and Bin $\mathrm{Hu}^{*}$

\author{
Affiliation: \\ Department of Materials Science and Engineering, University of Tennessee, Knoxville, Tennessee, \\ 37996, USA. \\ *Corresponding author: \\ Bin Hu, E-mail: bhu@utk.edu
}




\section{1: Experimental Methods}

Materials and Device fabrication. Formamidinium bromine (FABr, 99\%) and PEDOT:PSS (Clevious PVP AI 4083) were purchased from Xi'an Polymer Light Technology Corp (PLT). Tin (II) iodide ( $\left.\mathrm{SnI}_{2}, 99.999 \%\right)$, Tin (II) Flouride $\left(\mathrm{SnF}_{2}, 99 \%\right)$ and Polyethylenimine (PEI) (branched, $M=20,000)$ were purchased from Sigma Aldrich. All the materials were used as received. Devices studied in this work were fabricated with an inverted structure of ITO/PEDOT:PSS/perovskite/ $\mathrm{PC}_{61} \mathrm{BM} / \mathrm{PEI} / \mathrm{Ag}$. The precursor solutions for $\mathrm{FASnI}_{2} \mathrm{Br}$ were prepared by dissolving $\operatorname{SnI}_{2}(1.3 \mathrm{M})$ with $\mathrm{FABr}(1.0 \mathrm{M})$ in a mixture solvent of $\gamma$-butyrolactone (GBL): dimethyl sulfoxide (DMSO) (7:3, v/v). Then $10 \mathrm{~mol} \% \mathrm{SnF}_{2}$ was added or not to the solutions. The mixture solutions were kept in dark condition for at least 2 hours to get all compounds fully dissolved. The PEDOT: PSS thin films were spin-coated, from solution filtered with $0.22 \mu \mathrm{m}$ PVDF filter, on the pre-cleaned ITO/glass substrates at $4000 \mathrm{rpm}$ for $60 \mathrm{~s}$ and then annealed at $150{ }^{\circ} \mathrm{C}$ for $30 \mathrm{~min}$ on a hot plate in ambient environment. The perovskite precursor solutions were spin coated onto PEDOT: PSS film using a two-step spin-coating process at 1000 rpm for $10 \mathrm{~s}$ and $4000 \mathrm{rpm}$ for $47 \mathrm{~s}$, and $400 \mu \mathrm{L}$ of toluene was dropped onto the perovskite films as anti-solvent at the last $20^{\text {th }}$ second. These films were then annealed at $90^{\circ} \mathrm{C}$ for $20 \mathrm{~min}$. After cooling down, phenyl- $\mathrm{C}_{61}$-butyric acid methyl ester $\left(\mathrm{PC}_{61} \mathrm{BM}, 20 \mathrm{mg} / \mathrm{mL}\right.$ in chlorobenzene) was spin-cast on top of perovskite films at $2000 \mathrm{rpm}$ for $50 \mathrm{~s}$. The films are kept in dark for at least 30 min, and then an interface-modifying layer PEI ( $0.1 \mathrm{wt} \%$, in anhydride 2-Propanol) at $5000 \mathrm{rpm}$ for $60 \mathrm{~s}$. Finally, 90-nm-thick silver top electrodes were thermally evaporated on top of the film through metal mask under a vacuum of $2 \times 10^{-4} \mathrm{~Pa}$. The active area of the devices is $0.06 \mathrm{~cm}^{2}$.

Characterizations and Measurements. The I-V characteristics were recorded by using Keithley 2400 source meter under illumination of AM $1.5 \mathrm{G} 100 \mathrm{~mW} / \mathrm{cm}^{2}$ from Newport solar simulator calibrated by a silicon reference cell. The absorption spectra were recorded using UV3600 (Shimadzu, Japan). The external quantum efficiency (EQE) spectra were measured by a handmade system. The cells were subjected to monochromatic illumination (300 W Xe lamp passing through a monochromator and appropriate filters). The light intensity was calibrated by a standard Si biased photodetector (DET100A, $350 \mathrm{~nm}-1100 \mathrm{~nm}$, Thor Labs). The light beam was chopped at $230 \mathrm{~Hz}$ and the response of the cell was acquired by a Stanford Research SR830 lock-in amplifier. Magneto-photocurrent measurements were conducted by recording photocurrent (Jsc) as a function of magnetic field up to $\sim 900 \mathrm{mT}$ (parallel to device plane). The magnetic field was 
applied parallel to the device plane while using $488 \mathrm{~nm} \mathrm{CW}$ laser as a photoexcitation. Photocurrent was monitored by Keithley 2400 source meter under short-circuit condition upon switching the photoexcitation between linear and circular polarizations at same intensity (148 $\left.\mathrm{mW} / \mathrm{cm}^{2}\right)$ and wavelength(405nm). Essentially, as schematically shown in our previous work ${ }^{\text {Error! }}$ Bookmark not defined, the circularly polarized light was converted from linearly polarized $405 \mathrm{~nm} \mathrm{CW}$ laser beam perpendicularly through a $\lambda / 4$ wave plate at $45^{\circ}$. Also, a commercial silicon solar cell was used as the reference to ensure no change generated in light reflection with the optical path. Meanwhile, the light intensity dependent Jsc and Voc was measured with neutral density filters stacked on the devices to modify the light intensity. The C-f and C-V measurements were performed by using a dielectric spectrometer (Agilent, 4294A) with alternating voltage of $50 \mathrm{mV}$. 


\section{2: Supplementary figures}

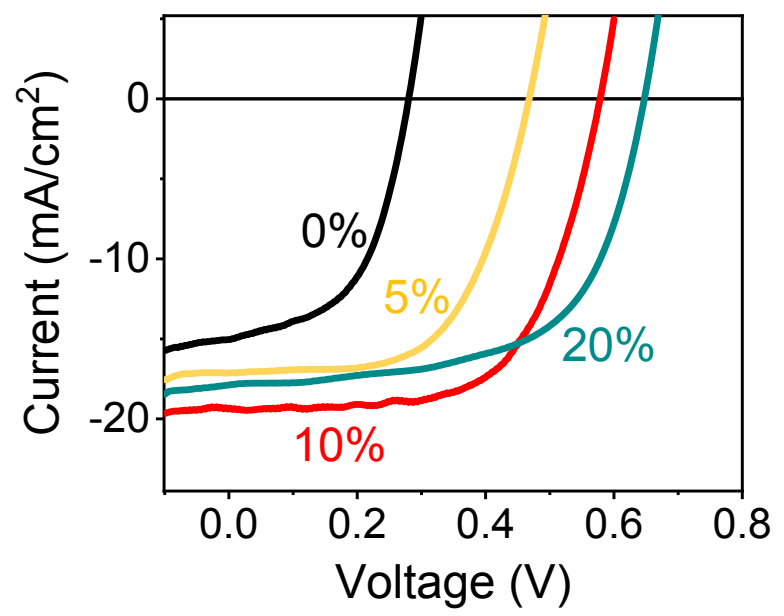

Figure S1. Current-voltage characteristics measured under 1 sun condition $\mathrm{FASnI}_{2} \mathrm{Br}$ perovskite solar cells ([ITO/PEDOT:PSS/FASnI $\left.\left.{ }_{2} \mathrm{Br} / \mathrm{PC}_{61} \mathrm{BM} / \mathrm{PEI} / \mathrm{Ag}\right]\right)$ with different doping concentration of $\mathrm{SnF}_{2}$.

Table S1. Photovoltaic parameters for $\mathrm{FASnI}_{2} \mathrm{Br}$ perovskite solar cells with different doping concentration of $\mathrm{SnF}_{2}$.

\begin{tabular}{ccccc}
\hline $\begin{array}{c}\text { Doping } \\
\text { concentration }\end{array}$ & $\mathrm{Jsc}\left(\mathrm{mA} / \mathrm{cm}^{2}\right)$ & Voc $(\mathrm{V})$ & Fill factor $(\%)$ & PCE $(\%)$ \\
\hline $0 \%$ & 14.87 & 0.27 & 51.5 & 2.07 \\
$5 \%$ & 17.13 & 0.47 & 59.5 & 4.79 \\
$10 \%$ & 19.82 & 0.60 & 64.0 & 7.61 \\
$20 \%$ & 17.89 & 0.65 & 61.4 & 7.10 \\
\hline
\end{tabular}



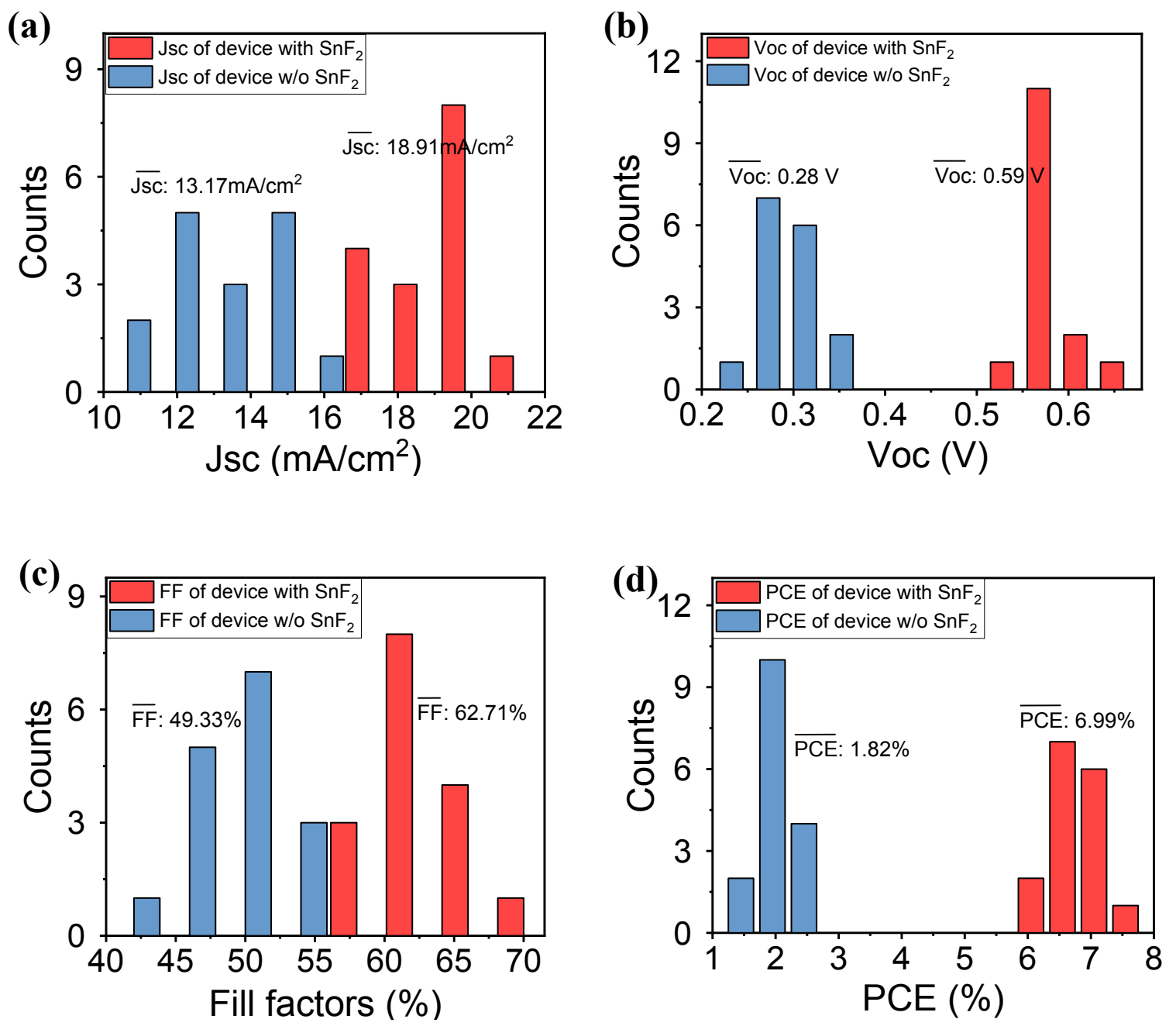

Figure S2. Statistical photovoltaic parameters for $\mathrm{FASnI}_{2} \mathrm{Br}$ perovskite solar cells with and without $\mathrm{SnF}_{2}$ doping (each with 16 cells). (a) Jsc, (b) Voc, (c) FF, (d) PCE. 


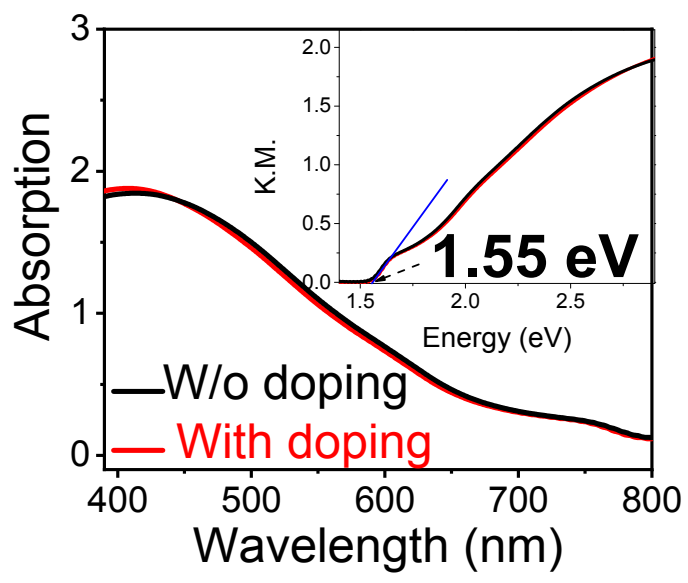

Figure S3. Optical absorption spectra for doped and undoped $\mathrm{FASnI}_{2} \mathrm{Br}$ perovskite films. 


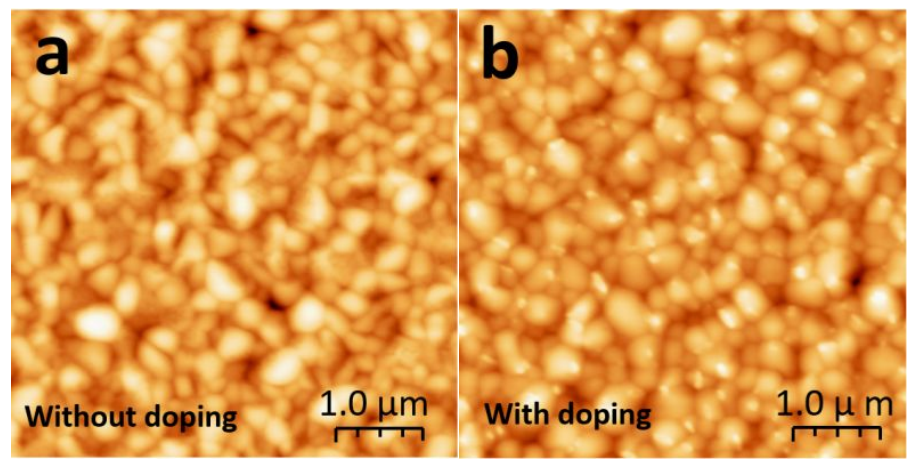

Figure S4. Atomic Force Microscopy (AFM) characteristics for doped and undoped Sn perovskite films. 